\title{
The Successive Ejection of Several Halo CMEs from NOAA AR. 652 July 2004, a Physical Study
}

\author{
Shahinaz Yousef ${ }^{1}$, Mostafa M. El-Nazer ${ }^{1}$ and Aisha Bebars ${ }^{2}$ \\ ${ }^{1}$ Astronomy\& Meteorology Dept, Faculty of Science, Cairo University, email: \\ shahinazyousef@yahoo.com \\ ${ }^{2}$ National Research Institute of Astronomy and Geophysics, Cairo, Egypt
}

\begin{abstract}
Although we are on the descending branch of solar cycle 23, a very strong active region, NOAA 652, crossed the solar visible hemisphere during the second half of July 2004. Its very large sunspot was of beta- gamma- delta type. This active region was a source of numerous $\mathrm{X}$-ray flares and coronal mass ejections. Being in a favorable position not far from the equator, it represented a threat to planet Earth particularly when near its central meridian passage.

Using EIT images, it was possible to locate the position of the 2004 July 25 CME ejection as dimming of several loops to the SW of AR 652. As usual, the CME lift up was accompanied by type IV burst and followed by type II burst and a proton flare.
\end{abstract}

Keywords. Sun: coronal mass ejections (CMEs), flares, particle emission

\section{The Ejection Conditions of The 2004 July 25 CME}

A list of coronal mass ejections (CMEs) produced by active region, NOAA 652 is given in ftp://lasco6.nascom.nasa.gov/pub/lasco/status/LASCO_CME_List_2004.

Since space is limited, only the July $25 \mathrm{CME}$ will be studied here. It was preceded by two brief X-ray flares. The sequence of events that lead and accompanied the CME are; a type IV burst, a M1.1 X-ray flare,a 1F chromospheric flare and a Type II radio burst respectively as seen below.

$\begin{array}{lllll}\text { Type } & \text { start } & \max & \text { end } & \text { location } \\ \text { C2.1 } & 13: 18 & 13: 25 & 13: 32 & \text { N04W29 } \\ \text { M2.2 } & 13: 37 & 13: 49 & 13: 55 & \text { N04W30 } \\ \text { M1.1 } & 14: 19 & 15: 14 \mathrm{UT} & 16: 43 & \text { N08W33 } \\ \text { 1F Flare } & 1433 & 1448 & 1643 & \text { N08W33 } \\ \text { Type IV/2 } & 1415 & / / / / & 1731 & \\ \text { Type II } & 1521 & / / / / & 1526 & \end{array}$

Shocks generated during the CMEs are detected as type II radio bursts while ejected plasmoids are observed as type IV bursts(Gopalswamy et al. 1999). The plasmoid was perhaps lifted over around the time of type IV start at 1415.

A front side halo CME is usually apparent in EIT images as dimming (Zhang et al. 2003). This dimming is actually apparent in fig. 1 images at 1348 and 1500. In the first image a faint bright loop seen first at 1326 was dimmed. The second important dimming of several coronal loops seen at 1500 is the actual site of CME ejection. However the CME was actually ejected earlier. It was first seen in C2 around 1430 and in C3 in SW limb at 1518 UT. Plane-of-sky speed at PA 145 was $1280 \mathrm{kms}^{-1}$ (ftp://lasco6.nascom.nasa.gov/pub/lasco/status/LASCO_CME_List_004) 

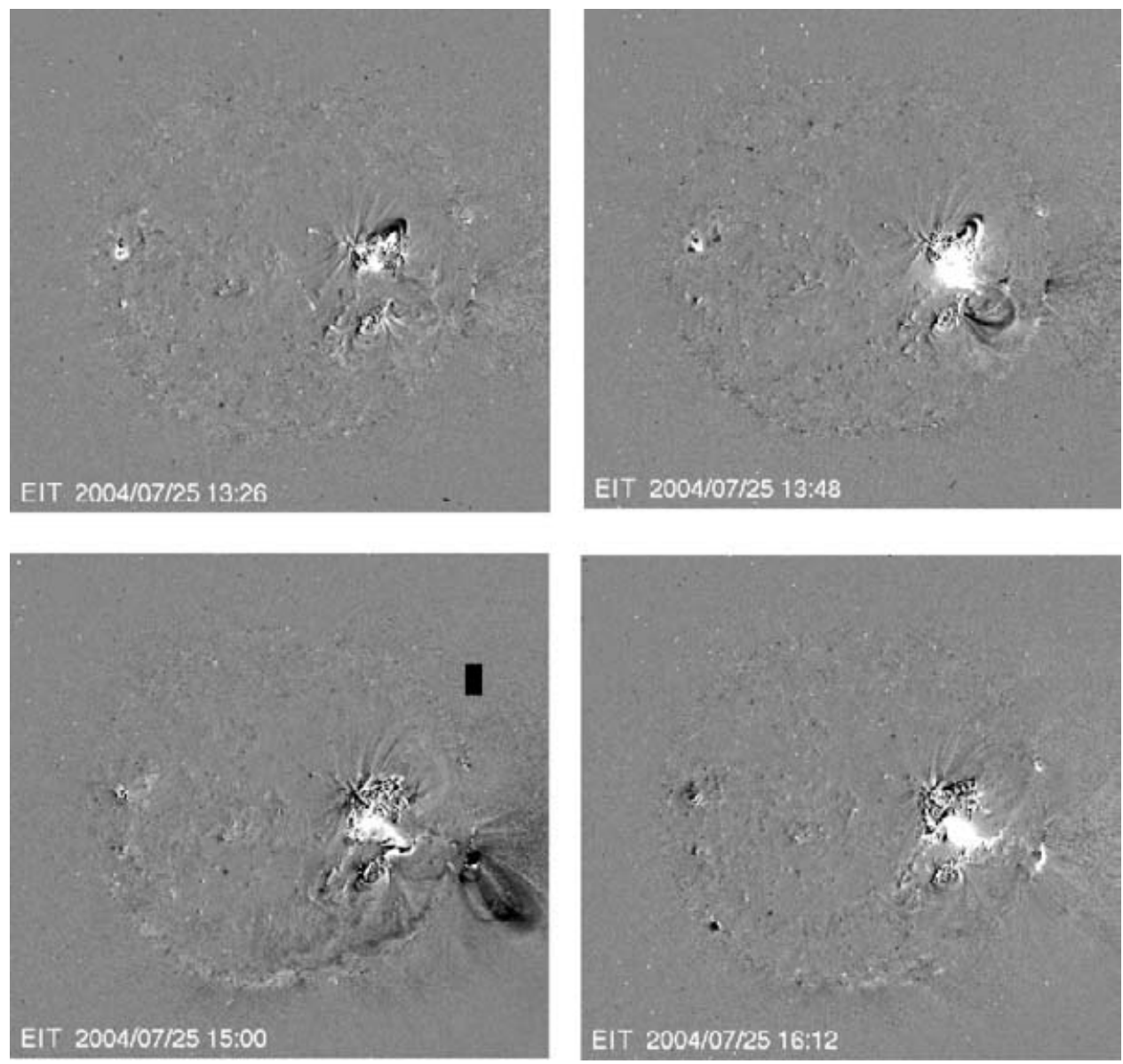

Figure 1. A sequence of EIT images showing the site of coronal mass ejection of 2004/07/25. Note the faint bright loop at 1326 that was dimmed on 1348. Several dimmed loops to the SW of AR NOAA 652 seen on 1500 image and disappeared later, are the most probable site of initiation of coronal mass ejection. The wave front to the SE of those dark loops is disturbed on 1500 due to type IV radio burst. Two C2.1 and M2.2 flares were near their maximums on 13.26 and 13.48 images respectively. Another M1.1 flare was going on during the images at 1500 and 1612 between the two ARs NOAA 652 and 653 and magnetic loops were seen later to connect them. Images are reproduced from ftp://ares.nrl.navy.mil/pub/lasco/halo/20040725/

\section{Conclusion}

The 25th of July 2004 CME was ejected from a site to the SW of ARs NOAA 652 and 653. Apparent dimming of several coronal loops in this location was evident in EIT images. The ejection was preceded by two brief X-ray flares. A type IV radio burst started four minutes ahead of a third long duration M1.1 X-ray flare accompanied the lift off of the CME. A type II radio wave and a proton flare followed.

\section{References}

Gopalswamy, N., Lazio, T. J. W., Kassim, N. E., and Erickson, W. C. 1999, BAAS, 194, 848

Zhang, J., Dere K. P., Howard, R. H., and Bothmer, V. 2003, ApJ 582, 520 